\title{
Symptom burden and medication use in adult sarcoma patients
}

\author{
Alexandre Chan • Eunice Lim • Terence Ng • \\ Vivianne Shih • Richard Quek • Yin Ting Cheung
}

Received: 31 August 2014 / Accepted: 17 November 2014 / Published online: 29 November 2014

(C) Springer-Verlag Berlin Heidelberg 2014

\begin{abstract}
Purpose Limited data are available on how symptom burden affects health-related quality of life (HRQoL) in patients with sarcoma. This study aims to describe the symptom burden, HRQoL, and medication use in adult sarcoma patients.

Methods A single-center, cross-sectional study was conducted, and 79 patients were evaluated using three tools: the Rotterdam Symptom Checklist (RSCL), the Beck Anxiety Inventory (BAI), and the Functional Assessment of Cancer Therapy scale - General (FACT-G). Patients' demographic and clinical information, medication history, and use of concomitant medications were recorded. The proportion of patients with clinically significant RSCL score for a particular symptom was compared with the percentage of patients receiving medication for that symptom.

Results The mean age was $57.3 \pm 15.2$ years, with majority of the patients diagnosed with stromal tumor $(46.8 \%)$, leiomyosarcoma (15.2\%), and liposarcoma (10.1\%). The most prevalent physical symptoms experienced were tiredness $(2.38 \pm 1.00)$, lack of energy $(2.04 \pm 1.02)$, and difficulty sleeping $(2.00 \pm 1.15)$. The most common psychological symptoms experienced were irritability $(1.92 \pm 1.01)$, worrying $(1.86 \pm 0.90)$, and anxiety $(1.68 \pm 0.74)$. Few $(6.3 \%)$ patients received hypnotics while $33.0 \%$ of patients reported difficulty sleeping. A proportion of patients $(27.9 \%)$ reported experiencing lack of appetite with only $1.3 \%$ received appetite stimulants.
\end{abstract}

A. Chan $(\bowtie) \cdot$ E. Lim $\cdot$ T. Ng $\cdot$ Y. T. Cheung

Department of Pharmacy, Faculty of Science, National University of Singapore, 18 Science 4, Block S4, Singapore 117543, Singapore e-mail: phaac@nus.edu.sg

\section{A. Chan • V. Shih}

Department of Oncology Pharmacy, National Cancer Centre

Singapore, Singapore, Singapore

R. Quek

Division of Medical Oncology, National Cancer Centre Singapore, Singapore, Singapore
Conclusion Adult sarcoma patients experience significant physiological and psychological symptom burden, which has a strong negative impact on HRQoL, with a number of physiological symptoms undertreated with pharmacotherapy.

Keywords Sarcoma $\cdot$ GIST $\cdot$ Adult $\cdot$ Symptom burden · Medication use $\cdot$ Cancer

\section{Introduction}

Sarcomas are cancers that arise from transformed cells of mesenchymal origin with heterogeneous differentiation. They are rare malignant tumors, with incidence rates ranging from 1.8 to 5.0 cases per 100,000 per year [1]. Most adult patients diagnosed with sarcoma present with large, aggressive tumors. Hence, these patients often suffer from debilitating disease- or treatment-related adverse effects and symptom burden [2]. Numerous studies have suggested that patients with sarcoma suffer from poorer health-related quality of life (HRQoL) at diagnosis and during treatment [3-5]. This is attributed to decreased physical and cognitive functioning, disease interference in family life, social and leisure activities, and an intense experience of disease- and treatment-related symptoms. In the literature, however, little is known about the effectiveness of symptom control using pharmacotherapy in patients with sarcoma. One study suggested that $53 \%$ of patients with soft tissue sarcoma (STS) experienced pain at the time of assessment, of whom more than half had pain that was described as inadequately controlled [6]. Another study has shown that pain (50-82 \%), dyspnea (20-40\%), and nausea and vomiting (18-22\%) were the most prevalent symptoms experienced by patients with locally advanced and metastatic STS [7]. Certain symptoms, such as dyspnea, are often undertreated [7]. As sarcoma patients receive complex systemic treatment, it may be challenging for these patients to experience complete symptom control, and inadequate control 
of disease or treatment-related symptoms may have a negative impact on patients' HRQoL [8].

Currently, there are limited data addressing the HRQoL in patients with sarcoma. This group of patients may suffer from significant symptom burden, and its impact on HRQoL has not been established. Further prospective HRQoL data could aid in decision-making for patients with sarcoma. In view of the scarcity of literature that evaluates the prevalence and severity of symptom burden of sarcoma patients, this study was designed to investigate the symptom burden experienced by adult sarcoma patients who were receiving treatment and to examine the relationship between symptom burden and HRQoL. This study also aimed to investigate the pattern of medication use for the control of patients' disease- and/or treatment-related symptoms. Through a better understanding of the symptom burden, HRQoL, and symptomatic medication use of the adult sarcoma patients, clinicians will be able to make informed clinical decisions to optimize therapeutic care.

\section{Methods}

\section{Study design and setting}

This was a cross-sectional study, conducted between October 2013 and February 2014 at the National Cancer Centre Singapore (NCCS). The NCCS is the largest ambulatory cancer center in Singapore that treats about $70 \%$ of all cancer patients in the country. This study was approved by the SingHealth Centralised Institutional Review Board. Informed consent was obtained from all participants prior to study enrollment.

\section{Patients}

Patients were recruited if they fulfilled the following inclusion criteria: (1) age 18 years and older at time of study entry, (2) confirmed histological diagnosis of sarcoma, (3) receiving therapy (either chemotherapy, radiotherapy, or surgery) at the point of the study, and (4) ability to understand English or Mandarin. Patients were excluded from the study if they were physically or mentally incapable of giving informed consent.

\section{Data collection procedure}

Patients' demographic and clinical information, such as age, gender, education level, sarcoma subtype and stage, Eastern Cooperative Oncology Group (ECOG) status, type of anticancer treatment (surgery, chemotherapy, targeted therapy, or radiation), and comorbidities were collected from in-house electronic databases. Patients' medication histories and concomitant use of medications (including complementary alternative medication) were also recorded. Social histories including smoking status, alcohol usage, and frequencies of exercise were captured in the demographic survey. Additional relevant clinical information was retrieved from a manual search of medical records. Missing data from hospital records were obtained from patients' primary care team and through verbal confirmation with patients.

A 30-min interview was conducted in either English or Mandarin with the aid of trained bilingual interviewers. The symptom burden and HRQoL of patients were assessed using three tools, namely the Rotterdam Symptom Checklist (RSCL), the Beck Anxiety Inventory (BAI), and the Functional Assessment of Cancer Therapy scaleGeneral (FACT-G).

\section{Endpoints/outcomes}

\section{Symptom burden}

The RSCL was used to evaluate adult sarcoma patients' symptom burden and their HRQoL. The term "symptom burden" can be defined as symptoms experienced by patients as a result of the disease itself or due to associated treatments [9]. The RSCL is a questionnaire with 23 items describing physiological symptom distress, 7 items describing psychological distress, 8 items describing the patient's activity level, and 1 item describing the patient's overall evaluation of life. The overall evaluation of life is on a seven-point Likert-type scale, while the other response categories are on a four-point Likert-type scale ("not at all," "a little," "quite a bit," and "very much"). In this study, symptoms that scored 3 and above were defined as clinically important symptoms to patients. A total physiological symptom burden score can be obtained from this method ranging from 23 to 92, and a total psychological symptom burden score can be obtained ranging from 7 to 28 . The activity level of patients can be measured on a scale of 0 to 24 , and the overall evaluation of life can be measured on a scale of -6 to 0 , a higher score signifying a higher evaluation of life. The scores of scales can be standardized by transforming raw scores into scores on a 100-point scale through the following equation: $\left[\frac{\text { Raw scale score }- \text { minimum raw score }}{\text { maximum-minimum score }} \times 100=\right.$ transformed score $]$ to facilitate interpretation and comparison between the physiological symptom domain and the psychological symptom domain. The RSCL is reliable as its internal consistency has been established (Cronbach's $\alpha>0.7$ ). It is also a validated tool to depict one's symptom burden and HRQoL based on correlation between scores in instrument (correlation coefficient (cc) $r>0.5$ between the items concerning overall evaluation of life and evaluation of physical function, psychological function, social function, and daily activities) and based on correlation with other measures (cc $r>0.5$ with Medical Outcome Study instrument, cc $r=0.74$ with Spielberger's State-Trait Anxiety scale) [10]. 
Patients' anxiety characteristics were evaluated using the BAI, which is a 21-item self-report inventory that assesses the emotional, physiological, and cognitive symptoms of anxiety experienced by patients in the past month. Each item on the BAI is a simple description of an anxiety-related symptom in one of its four expressed aspects: (1) subjective (e.g., "unable to relax"), (2) neurophysiologic (e.g., "numbness or tingling"), (3) autonomic (e.g., "feeling hot"), or (4) panic-related (e.g., "fear of losing control") [11]. Each symptom item would be rated as follows: "not at all," "mild," "moderate," or "severe." The BAI is designed to discriminate between anxiety and depression [12] and is reliable as it has high internal consistency (Cronbach's $\alpha=0.92$ ). It is also a validated tool for measuring anxiety, as its correlation score with the revised Hamilton Anxiety Rating Scale is $r=0.51$, whereas its correlation with the Hamilton Depression Rating Scale is $r=0.25$ [13]. The values for each item are summed, yielding a total score for all 21 symptoms that can range between 0 and 63 points. A total score of 0-7 is interpreted as "minimal", 8-15 as mild, 16-25 as moderate, and 26-63 as "severe" level of anxiety [14].

\section{Health-related quality of life}

The FACT-G was used to assess the impact of patients' HRQoL, as it provides a better insight on their physical, social/familial, emotional, and functional well-being. FACT$\mathrm{G}$ was selected because of its availability in multiple languages, its ease of administration, and the relatively short amount of time required for completion. It is a reliable tool, as it has high internal consistency (Cronbach's $\alpha=0.86$ ) and has been validated in patients with cancer. The FACT-G total score ranges from 0 to 108 , with higher scores signifying a higher HRQoL $[15,16]$.

\section{Medication use}

Through patient interview, detailed medical histories were recorded in the data collection form, which included name and class of medications, indications, doses, frequencies, route of administration, the dates of initiation and discontinuation of medications, and patient compliance. In addition, use of complementary alternative medications such as traditional Chinese medication (TCM); herbal, over-the-counter, or vitamin products; or medications prescribed by other healthcare professionals were also assessed. In this study, systemic anticancer treatment is defined as the use of chemotherapy or targeted therapy.

\section{Statistical analysis}

Patient demographics and baseline characteristics were reported in frequencies and percentages. Descriptive statistics were used to summarize the prevalence of physiological and psychological burden amongst patients, along with their degree of activity level impairment and overall valuation of life. Descriptive statistics were also used to summarize patients' BAI and FACT-G domain scores and total scores. Pearson's correlation analysis was conducted to determine the association between symptom burden scores and HRQoL scores. An independent sample $T$ test was used to investigate the differences in the severity of symptom burden experienced by different patient subgroups. Data analysis was conducted using IBM's Statistical Package for the Social Sciences, version 21.

\section{Results}

Patient demographics, clinical characteristics, and health-related quality of life

A total of 81 patients fulfilled the inclusion criteria. Two patients $(2.5 \%)$ declined participation, and 79 were included in the final analysis (response rate $97.5 \%$ ). Slightly over half of the patients were male $(58.2 \%)$, Chinese $(83.5 \%)$, and above 60 years old (50.6\%) (Table 1). The mean age was $57.3 \pm 15.2$ years, with $91.1 \%$ of the patients residing with a caregiver. Most of the patients $(87.3 \%)$ had an ECOG status of 0 , and a significant portion of them (49.4\%) had other comorbidities, with hypertension $(35.4 \%)$ and hyperlipidemia $(24.1 \%)$ being the most common. Majority (46.8 \%) of the patients were diagnosed with gastrointestinal stromal tumor (GIST), followed by leiomyosarcoma (15.2\%) and liposarcoma (10.1\%) (Table 2).

In terms of HRQoL, the mean total FACT-G score was 81.2 $( \pm 17.7)$. The mean scores for the four subdomains were as follows: social/familial well-being $(22.4 \pm 4.4)$, physical wellbeing (20.4 \pm 7.0$)$, emotional well-being (18.5 \pm 4.8$)$, and functional well-being (19.9 \pm 6.2$)$.

Symptom burden

The mean scores for the RSCL symptom and domain scores are summarized in Table 3 . The mean $( \pm \mathrm{SD})$ physiological symptoms score was $39.2( \pm 10.6)$, and the mean psychological symptoms score was $11.7( \pm 3.6)$. Upon transformation to a 100-point scale, it was found that the patient population had comparable intensity of physiological symptom burden $(23.5 \pm 15.4)$ and psychological symptom burden $(21.9 \pm 17.2)$. The most common physiological symptoms experienced were tiredness $(2.38 \pm 0.99)$, lack of energy (2.04 \pm 1.02$)$, and difficulty sleeping (2.00 \pm 1.09$)$, while the least common physiological symptoms experienced 
Table 1 Baseline demographic patient information $(n=79)$

\begin{tabular}{|c|c|c|}
\hline Demographic information & Total frequency & $\%$ \\
\hline Age (years) mean \pm SD & $57.3 \pm 15.2$ & \\
\hline $18-34$ & 8 & 10.1 \\
\hline $35-69$ & 54 & 68.4 \\
\hline$\geq 70$ & 17 & 21.5 \\
\hline \multicolumn{3}{|l|}{ Gender } \\
\hline Male & 46 & 58.2 \\
\hline Female & 33 & 41.8 \\
\hline \multicolumn{3}{|l|}{ Race } \\
\hline Chinese & 66 & 83.5 \\
\hline Malay & 7 & 8.9 \\
\hline Indian & 2 & 2.5 \\
\hline Others & 4 & 5.1 \\
\hline \multicolumn{3}{|l|}{ Marital status } \\
\hline Single & 10 & 12.7 \\
\hline Married & 65 & 82.3 \\
\hline Divorced/widowed & 4 & 5.0 \\
\hline \multicolumn{3}{|l|}{ Living alone } \\
\hline Yes & 7 & 8.9 \\
\hline \multicolumn{3}{|l|}{ How disease was diagnosed } \\
\hline Patient experienced symptoms & 66 & 83.5 \\
\hline Health screening & 9 & 11.4 \\
\hline Imaging due to other disease & 4 & 5.1 \\
\hline \multicolumn{3}{|l|}{ Education status } \\
\hline None & 7 & 8.9 \\
\hline Primary & 16 & 20.3 \\
\hline Secondary & 26 & 32.9 \\
\hline Preuniversity & 15 & 19.0 \\
\hline Graduate/postgraduate & 15 & 19.0 \\
\hline \multicolumn{3}{|l|}{ Employment status } \\
\hline Unemployed & 23 & 29.1 \\
\hline Freelance & 1 & 1.3 \\
\hline Employed & 30 & 38.0 \\
\hline Student & 3 & 3.8 \\
\hline Retired & 19 & 24.1 \\
\hline On long-term medical leave & 3 & 3.8 \\
\hline \multicolumn{3}{|l|}{ Body mass index $\left(\mathrm{kg} / \mathrm{m}^{2}\right)$} \\
\hline$<18.5$ & 6 & 7.6 \\
\hline $18.5-24.99$ & 50 & 63.3 \\
\hline$\geq 25.00$ & 23 & 29.1 \\
\hline \multicolumn{3}{|l|}{ Smoking status } \\
\hline Current smoker & 13 & 16.5 \\
\hline \multicolumn{3}{|l|}{ Alcohol status } \\
\hline Current drinker & 5 & 6.3 \\
\hline \multicolumn{3}{|l|}{ Exercise status } \\
\hline Yes & 34 & 43.0 \\
\hline
\end{tabular}

were headache $(1.43 \pm 0.76)$, shivering $(1.35 \pm 0.75)$, and vomiting $(1.29 \pm 0.64)$. Among the physiological symptoms, tiredness $(46.7 \%)$, difficulty sleeping $(33.0 \%)$, and lack of appetite (30.4\%) were perceived as most clinically important to patients. The numbers of clinically significant physiological and psychological symptoms were $4.38 \pm 4.25$ and $1.00 \pm 1.53$ respectively.

On the other hand, the most common psychological symptoms experienced were irritability $(1.92 \pm 1.01)$, worrying $(1.86 \pm 0.90)$, and anxiety $(1.68 \pm 0.74)$ while the least common psychological symptoms were depressed mood $(1.51 \pm 0.77)$, despairing about the future $(1.51 \pm 0.77)$, and nervousness $(1.53 \pm 0.66)$. Among the psychological symptoms, irritability (26.6\%), worrying $(21.5 \%)$, and anxiety $(14.0 \%)$ were perceived as most clinically important to patients. The overall activity level impairment was low, with a mean score of $4.29( \pm 5.96)$, and the overall evaluation of life had a mean score of $-1.91( \pm 1.49)$.

\section{Correlation analysis}

The RSCL physiological symptom scores and psychological symptom scores were negatively correlated with FACT-G total scores $\left(r_{\mathrm{sp}}=-0.75\right.$ and $\left.r_{\mathrm{sp}}=-0.67\right)$ and RSCL overall evaluation of life $\left(r_{\mathrm{sp}}=-0.59\right.$ and $\left.r_{\mathrm{sp}}=-0.53\right)$ (Table 4). In addition, the numbers of clinically significant physiological and psychological symptoms are also negatively correlated with FACT-G total scores $\left(r_{\mathrm{sp}}=-0.67\right.$ and $\left.r_{\mathrm{sp}}=-0.57\right)$. RSCL physiological symptom burden scores were strongly correlated with BAI scores $\left(r_{\mathrm{sp}}=0.72\right)$, whereas the correlation between psychological symptom burden scores and BAI scores was weaker $\left(r_{\mathrm{sp}}=0.56\right)$. In comparison, RSCL activity level impairment scores had a lower correlation with BAI and with FACTG scores $\left(r_{\mathrm{sp}}=0.36\right.$ and $\left.r_{\mathrm{sp}}=-0.57\right)$. Finally, age and cancer stage did not appear to correlate with the physiological and psychological symptom burden scores or with activity level impairment scores or FACT-G scores.

\section{Subgroup analysis}

Subgroup analysis revealed that non-GIST patients had higher physiological symptom burden scores when compared to GIST patients ( 41.3 vs. $36.8, p=0.048$ ) (Table 5). They also had a statistically significantly higher psychological symptom burden scores (12.3 vs. 10.7, $p=0.045)$, and the results were statistically significant $(p=0.045)$. Non-GIST patients also had higher levels of activity impairment (5.7 vs. 2.7, $p=0.02$ ), higher BAI scores (13.4 vs. 7.7, $p=0.004)$, and lower FACT-G scores (76.4 vs. 86.6, $p=0.008$ ). There was no statistically significant difference between the symptom burden scores, activity level impairment scores, BAI scores, and HRQoL scores of patients with versus without comorbidities, as well as patients receiving systemic anticancer therapies versus non-systemic anticancer therapies. 
Table 2 Clinical and medical patient information $(n=79)$

\begin{tabular}{|c|c|c|}
\hline Clinical information & Total frequency & $\%$ \\
\hline \multicolumn{3}{|l|}{ Type of sarcoma } \\
\hline Angiosarcoma & 2 & 2.5 \\
\hline Chondrosarcoma & 2 & 2.5 \\
\hline Ewing sarcoma & 2 & 2.5 \\
\hline Fibrosarcoma & 4 & 5.1 \\
\hline GIST & 37 & 46.8 \\
\hline Leiomyosarcoma & 12 & 15.2 \\
\hline Liposarcoma & 8 & 10.1 \\
\hline Osteosarcoma & 3 & 3.8 \\
\hline Rhabdomyosarcoma & 3 & 3.8 \\
\hline Miscellaneous & 6 & 7.6 \\
\hline \multicolumn{3}{|l|}{ Time from diagnosis } \\
\hline $0-6$ months & 17 & 21.5 \\
\hline$>6$ months -1 year & 15 & 19.0 \\
\hline$>1-3$ years & 12 & 15.2 \\
\hline$>3-5$ years & 15 & 19.0 \\
\hline$>5-10$ years & 17 & 21.5 \\
\hline$>10$ years & 3 & 3.8 \\
\hline \multicolumn{3}{|l|}{ Previous treatment modes } \\
\hline Chemotherapy & 61 & $77.2^{\mathrm{a}}$ \\
\hline Radiotherapy & 22 & $27.8^{\mathrm{a}}$ \\
\hline Surgery & 67 & $84.8^{\mathrm{a}}$ \\
\hline \multicolumn{3}{|l|}{ Current treatment status } \\
\hline Receiving chemotherapy & 15 & 19.0 \\
\hline Receiving radiotherapy & 4 & 5.1 \\
\hline Receiving targeted therapy & 37 & 46.8 \\
\hline Imatinib & 31 & 39.2 \\
\hline Sunitinib & 4 & 5.1 \\
\hline Regorafenib & 1 & 1.3 \\
\hline Pazopanib & 1 & 1.3 \\
\hline Preoperation & 5 & 6.3 \\
\hline Postoperation & 18 & 22.8 \\
\hline \multicolumn{3}{|l|}{ Cancer stage } \\
\hline I & 13 & 16.5 \\
\hline II & 18 & 22.3 \\
\hline III & 29 & 36.7 \\
\hline IV & 19 & 24.1 \\
\hline \multicolumn{3}{|l|}{ ECOG status } \\
\hline 0 & 69 & 87.3 \\
\hline 1 & 7 & 8.9 \\
\hline 2 & 1 & 1.3 \\
\hline 3 & 0 & 0 \\
\hline 4 & 2 & 2.5 \\
\hline \multicolumn{3}{|l|}{ Comorbidities } \\
\hline Presence & 39 & 49.4 \\
\hline Hypertension & 28 & $35.4^{\mathrm{a}}$ \\
\hline Hyperlipidemia & 19 & $24.1^{\mathrm{a}}$ \\
\hline Diabetes mellitus & 8 & $10.1^{\mathrm{a}}$ \\
\hline Hepatitis B & 7 & $8.9^{\mathrm{a}}$ \\
\hline
\end{tabular}

Table 2 (continued)

\begin{tabular}{lcc}
\hline Clinical information & Total frequency & $\%$ \\
\hline Asthma & 3 & $3.8^{\mathrm{a}}$ \\
Others & 5 & $6.3^{\mathrm{a}}$ \\
Receipt of any alternative medicine or therapies & \\
Yes & 49 & 62.0 \\
TCM & 14 & $17.7^{\mathrm{a}}$ \\
Vitamin supplements & 11 & $13.9^{\mathrm{a}}$ \\
OTC products & 3 & $3.8^{\mathrm{a}}$ \\
Prescribed by others & 8 & $10.1^{\mathrm{a}}$ \\
\hline
\end{tabular}

GIST gastrointestinal stromal tumor, ECOG status Eastern Cooperative Oncology Group performance status, TCM traditional Chinese medication, OTC products over-the-counter medication products

${ }^{\text {a }}$ Percentage out of 79 patients and does not add up to $100 \%$

Medication use

A considerable proportion of patients (38.0\%) were receiving chronic medications (Table 6). Sixteen patients $(20.3 \%)$ were prescribed medications indicated for pain, $15(19.0 \%)$ were prescribed medications indicated for constipation, and 8 $(10.1 \%)$ received antiemetics. Six patients $(7.6 \%)$ received medications for relief of neuropathic pain, and five $(6.3 \%)$ received medication for dry/sore eyes. Additionally, five patients $(6.3 \%)$ were consuming sedatives/hypnotics, and three $(3.8 \%)$ were prescribed medications for anxiety/ depression. Only $1.3 \%$ of the patients received medication indicated for diarrhea and lack of appetite.

\section{Discussion}

To our best of knowledge, this is the first study that evaluates the impact of symptom burden on HRQoL among adult patients with sarcoma. The results of this study demonstrated that this group of patients experienced significant physiological and psychological symptom burden and that a higher symptom burden was associated with poorer HRQoL. This is consistent with numerous studies that have shown a strong relationship between symptom burden and HRQoL in other cancer types $[17,18]$. All in all, these findings imply that it is important to provide adequate control of both physiological and psychological symptoms in order to improve sarcoma patients' HRQoL.

The most important physiological symptoms reported were tiredness, lack of energy, and difficulty sleeping. This differs from another study conducted by Gough et al. [7], which showed that the most common symptoms experienced by sarcoma patients with locally advanced and metastatic STS were pain, dyspnea, and nausea and vomiting. This difference 
Table 3 Rotterdam Symptom Checklist scores $(n=79)$

\begin{tabular}{|c|c|c|}
\hline (Theoretical score range) & $\begin{array}{l}\text { Mean } \pm \text { standard } \\
\text { deviation (SD) }\end{array}$ & $\begin{array}{l}\% \text { Perceived as clinically } \\
\text { important }\end{array}$ \\
\hline Physiological symptom domain (23-92) & $39.2 \pm 10.6$ & \\
\hline Physiological symptom domain $(0-100)$ & $23.5 \pm 15.4$ & \\
\hline Tiredness $(0-4)$ & $2.38 \pm 0.99$ & 46.8 \\
\hline Lack of energy & $2.04 \pm 1.02$ & 27.9 \\
\hline Difficulty sleeping & $2.00 \pm 1.09$ & 33.0 \\
\hline Sore muscles & $1.92 \pm 0.92$ & 22.8 \\
\hline Loss of hair & $1.90 \pm 1.08$ & 25.3 \\
\hline Lack of appetite & $1.85 \pm 1.00$ & 30.4 \\
\hline Dry mouth & $1.84 \pm 0.99$ & 24.1 \\
\hline Difficulty concentrating & $1.78 \pm 0.86$ & 17.8 \\
\hline Low-back pain & $1.78 \pm 0.99$ & 25.3 \\
\hline Shortness of breath & $1.71 \pm 0.85$ & 17.7 \\
\hline Constipation & $1.71 \pm 0.98$ & 19.0 \\
\hline Abdominal ache & $1.70 \pm 0.90$ & 19.0 \\
\hline Diarrhea & $1.63 \pm 0.89$ & 15.2 \\
\hline Acid indigestion & $1.63 \pm 0.89$ & 17.8 \\
\hline Burning/sore eyes & $1.63 \pm 0.85$ & 14.0 \\
\hline Decreased sexual interest & $1.53 \pm 0.81$ & 12.7 \\
\hline Tingling hands or feet & $1.53 \pm 0.81$ & 10.2 \\
\hline Dizziness & $1.53 \pm 0.79$ & 8.9 \\
\hline Sore mouth/pain when swallowing & $1.51 \pm 0.81$ & 12.7 \\
\hline Nausea & $1.49 \pm 0.90$ & 15.2 \\
\hline Headache & $1.43 \pm 0.76$ & 8.9 \\
\hline Shivering & $1.35 \pm 0.75$ & 8.9 \\
\hline Vomiting & $1.29 \pm 0.64$ & 5.0 \\
\hline Psychological symptom distress (7-28) & $11.6 \pm 3.6$ & \\
\hline Psychological symptom domain $(0-100)$ & $21.9 \pm 17.2$ & \\
\hline Irritability $(0-4)$ & $1.92 \pm 1.01$ & 26.6 \\
\hline Worrying & $1.86 \pm 0.90$ & 21.5 \\
\hline Anxiety & $1.68 \pm 0.74$ & 14.0 \\
\hline Tension & $1.54 \pm 0.73$ & 11.4 \\
\hline Nervousness & $1.53 \pm 0.66$ & 8.9 \\
\hline Depressed mood & $1.51 \pm 0.77$ & 8.9 \\
\hline Despairing about the future & $1.51 \pm 0.77$ & 8.9 \\
\hline Activity level impairment (0-24) & $4.29 \pm 5.96$ & \\
\hline Overall evaluation of life $(-6-0)$ & $-1.91 \pm 1.49$ & \\
\hline
\end{tabular}

Table 4 Correlation between RSCL, BAI, and FACT-G scores of adult sarcoma patients $(n=79)$

\begin{tabular}{|c|c|c|c|c|c|}
\hline & FACT-G total scores & RSCL overall evaluation of life & BAI total scores & Age & Cancer stage \\
\hline RSCL physiological symptom scores & $-0.75^{* *}$ & $-0.59 * *$ & $0.72 * *$ & -0.01 & 0.17 \\
\hline RSCL psychological symptom scores & $-0.67 * *$ & $-0.53 * *$ & $0.56^{* *}$ & -0.15 & 0.08 \\
\hline RSCL activity level impairment & $-0.57 * *$ & $-0.53 * *$ & $0.36^{* *}$ & -0.03 & 0.01 \\
\hline BAI total scores & $-0.64 * *$ & $-0.48 * *$ & - & -0.09 & 0.05 \\
\hline FACT-G total scores & - & $0.71 * *$ & - & 0.10 & -0.12 \\
\hline
\end{tabular}

**Statistically significant at the 0.01 level (two-tailed) 
Table 5 Subgroup comparison $(n=79)$

\begin{tabular}{|c|c|c|c|}
\hline Comparison groups & Mean \pm SD & Mean \pm SD & $p$ value \\
\hline Non-GIST versus GIST & Non-GIST $(n=42)$ & GIST $(n=37)$ & \\
\hline RSCL physiological symptom domain & $41.3 \pm 12.7$ & $36.8 \pm 7.0$ & $0.048 *$ \\
\hline RSCL psychological symptom domain & $12.3 \pm 4.3$ & $10.7 \pm 2.6$ & $0.045^{*}$ \\
\hline RSCL activity level impairment & $5.7 \pm 6.8$ & $2.7 \pm 4.3$ & $0.02 *$ \\
\hline RSCL overall evaluation of life & $-2.1 \pm 1.7$ & $-1.7 \pm 123$ & 0.30 \\
\hline BAI total score & $13.4 \pm 10.6$ & $7.7 \pm 5.7$ & $0.004 *$ \\
\hline FACT-G total score & $76.4 \pm 19.7$ & $86.6 \pm 13.3$ & $0.008^{*}$ \\
\hline With versus without comorbidities & With $(n=39)$ & Without $(n=40)$ & \\
\hline RSCL physiological symptom domain & $38.8 \pm 9.9$ & $39.6 \pm 11.4$ & 0.73 \\
\hline RSCL psychological symptom domain & $10.9 \pm 3.4$ & $12.2 \pm 3.8$ & 0.10 \\
\hline RSCL activity level impairment & $4.5 \pm 5.8$ & $4.1 \pm 6.2$ & 0.72 \\
\hline RSCL overall evaluation of life & $-1.8 \pm 1.4$ & $-2.0 \pm 1.6$ & 0.59 \\
\hline BAI total score & $10.3 \pm 8.4$ & $11.2 \pm 9.8$ & 0.66 \\
\hline FACT-G total score & $81.4 \pm 15.3$ & $80.9 \pm 19.9$ & 0.90 \\
\hline Systemic versus non-systemic anticancer therapies & Systemic $(n=52)$ & Non-systemic $(n=27)$ & \\
\hline RSCL physiological symptom domain & $38.3 \pm 11.7$ & $39.7 \pm 10.1$ & 0.58 \\
\hline RSCL psychological symptom domain & $11.41 \pm 3.8$ & $11.6 \pm 3.6$ & 0.80 \\
\hline RSCL activity level impairment & $3.44 \pm 5.1$ & $4.7 \pm 6.4$ & 0.37 \\
\hline RSCL overall evaluation of life & $2.59 \pm 1.4$ & $3.1 \pm 1.5$ & 0.17 \\
\hline BAI total score & $11.5 \pm 10.7$ & $10.4 \pm 8.2$ & 0.60 \\
\hline FACT-G total score & $82.7 \pm 15.9$ & $80.4 \pm 18.6$ & 0.59 \\
\hline
\end{tabular}

${ }^{*} p \leq 0.05$, statistically significant

in results may be due to variations in the study design and patient populations. This study included sarcoma patients with different cancer stages (only $24.1 \%$ are stage 4 patients) and current treatment statuses, whereas Gough et al. included only patients who were considered for palliative chemotherapy. Likewise, this study included GIST patients, whereas Gough et al. excluded GIST patients. GIST patients often receive imatinib as targeted therapy, which is associated with few side effects and provides a long-term survival benefit as compared to other treatment modalities. Hence, the symptom burden

Table 6 Indications of medications used by patients with sarcoma $(n=79)$

\begin{tabular}{lc}
\hline Indications & Patients receiving medications (\%) \\
\hline Chronic medications & $30(38.0)$ \\
Pain & $16(20.3)$ \\
Constipation & $15(19.0)$ \\
Nausea/vomiting & $8(10.1)$ \\
Neuropathic pain & $6(7.6)$ \\
Dry/sore eyes & $5(6.3)$ \\
Insomnia & $5(6.3)$ \\
Anxiety/depression & $3(3.8)$ \\
Diarrhea & $1(1.3)$ \\
Lack of appetite & $1(1.3)$ \\
\hline
\end{tabular}

experienced by the two patient populations may be different. It may also be inferred that patients experience different intensities and severities of symptom burden at different cancer stages, across different cancer types and with different treatments.

The most important psychological symptoms reported were irritability, worrying, and anxiety. It was also observed that a significant proportion of patients $(27.9 \%)$ experienced a moderate to severe degree of anxiety. Studies have shown that anxiety is generally higher at the onset of the cancer diagnosis $[19,20]$, and this was similarly reported in our study whereby one third of patients experienced at least a moderate degree of anxiety in which almost $40 \%$ of patients were newly diagnosed with sarcoma within 1 year from the beginning of the study. Correlation analysis also demonstrated that anxiety and psychological symptom domain scores had a strong negative correlation with patients' HRQoL, signifying that a reduction in patients' anxiety levels and psychological symptoms may lead to better HRQoL. This result is consistent with that of other studies and highlights the need to address the psychosocial domains across the cancer trajectory of patients, which would translate into improved HRQoL and outcomes for patients $[21,22]$. In contrast, clinical factors that are often perceived to affect HRQoL, such as the degree of activity level impairment, age, and cancer progression, did not affect patients' HRQoL score. Hence, it is suggestive that symptom 
burden strongly impacts the HRQoL of patients with sarcoma compared to other factors.

In addition to the identification of symptoms that commonly present in patients with sarcoma, it is also crucial to ensure that these symptoms are well controlled. Similarly to other studies, a significant proportion of these patients did not receive adequate management of pain and dyspnea $[7$, 23-25]. In addition, a substantial number were still suffering from clinically significant pain and shortness of breath. Possible explanations provided in literature include patients' underreporting of symptoms to clinicians, underutilization of medications, and a lack of evidence-based guidelines for the treatment of dyspnea [23, 24]. There are also several studies in the literature that highlight the undertreatment of insomnia in cancer patients, which may negatively affect HRQoL [26-28]. We note that although a significant portion (33\%) of patients reported difficulty sleeping on the RSCL, a small fraction $(6.3 \%)$ of patients were prescribed hypnotics. Evidence suggests that the treatment of insomnia can improve cancerrelated fatigue and related symptoms and consequently improve overall health and quality of life [26]. While there is a vast amount of evidence suggesting that clinicians should promote the use of hypnotic agents in patients with cancer, concerns including addiction and overutilization of hypnotics remain a concern in the medical community $[26,29,30]$. This is clinically relevant, as a significant proportion of patients in this study were localized GIST patients receiving adjuvant imatinib for chronic usage. Finally, almost one third of the patients reported a lack of appetite; however, very few patients received pharmacological therapies such as appetite stimulants. Although the use of megestrol acetate for appetite stimulation in cachexic patients with cancer is recommended $[25,31]$, the use of megestrol acetate is not routine in practice, as the amount of weight gain may not be clinically significant $[32,33]$ and numerous reports have suggested that megestrol acetate can cause secondary adrenal suppression and increased risk of thromoembolism in patients with cancer [34-36]. Therefore, it is not surprising that many patients did not receive pharmacological therapies to manage poor appetite. Due to potential side effects and insufficient evidence, non-pharmacological strategies, such as dietary changes, are more commonly employed in clinical practice. In summary, clinicians face a number of challenges in offering appropriate and adequate pharmacotherapy to address symptom burden in patients with sarcoma, which could be related to the limited number of strategies available, in addition to patients' willingness to report their degree of symptoms and their perceived safety profile of these therapies. As pharmacotherapy strategies are not always available, clinicians should always consider using non-pharmacological strategies for management of these symptoms.

This study had a few limitations. First, due to the crosssectional nature of this study, it was not possible to determine the change in patients' symptom burden or patients' anxiety characteristics after medication use. To evaluate the effectiveness of symptomatic medications on HRQoL, a longitudinal study design may be warranted. Although the RSCL provided a reasonable measure of overall symptom burden in patients, it did not provide specific details (such as onset and duration) of the symptoms experienced. More comprehensive tools should be incorporated in future studies to examine the characteristics and presentation of symptoms in detail. A significant proportion was users of complementary and alternative medicines. However, we do not know whether patients were using alternative therapies to manage their symptom burden. Finally, results may also be affected due to the aforementioned heterogeneity in clinical factors. However, due to the rarity of sarcoma, our patient population was representative of the patients that are frequently seen in daily clinical practice.

\section{Conclusion}

Adult patients with sarcoma experience a significant physiological and psychological symptom burden that has a strong negative impact on HRQoL. This includes physical symptoms such as tiredness, difficulty sleeping, and lack of appetite and psychological symptoms such as irritability, worrying, and anxiety. A number of symptoms, including pain, dyspnea, insomnia, and lack of appetite were undertreated among our patients. Future studies should be conducted to evaluate the impact of symptomatic medication use and symptom control on adult sarcoma patients' HRQoL.

Acknowledgments We would like to acknowledge the National University of Singapore Final Year Project grant and Terry Fox Foundation (NRFCB12131) for funding this study. We would also like to thank Ms. Yanxiang Gan for her assistance in this study.

Conflict of interest The authors have no conflicts of interest that are directly relevant to the content of this study.

\section{References}

1. Wibmer $C$ et al (2010) Increasing incidence rates of soft tissue sarcomas? A population-based epidemiologic study and literature review. Ann Oncol 21(5):1106-1111

2. Gapstur RL (2007) Symptom burden: a concept analysis and implications for oncology nurses. Oncol Nurs Forum 34(3):673-680

3. Paredes T et al (2011) Quality of life of sarcoma patients from diagnosis to treatments: predictors and longitudinal trajectories. Eur J Oncol Nurs 15(5):492-499

4. Speechley KN et al (2006) Health-related quality of life among child and adolescent survivors of childhood cancer. J Clin Oncol 24(16): 2536-2543 
5. Guest JF et al (2013) Utility values for advanced soft tissue sarcoma health states from the general public in the United Kingdom. Sarcoma 2013:863056

6. Kuo PY et al (2011) The prevalence of pain in patients attending sarcoma outpatient clinics. Sarcoma 2011:813483

7. Gough NJ et al (2011) Symptom burden, survival and palliative care in advanced soft tissue sarcoma. Sarcoma 2011:325189

8. Miaskowski C et al (2006) Subgroups of patients with cancer with different symptom experiences and quality-of-life outcomes: a cluster analysis. Oncol Nurs Forum 33(5):E79-E89

9. Cleeland CS (2007) Symptom burden: multiple symptoms and their impact as patient-reported outcomes. J Natl Cancer Inst Monogr 37: $16-21$

10. de Haes JCJM, Olschewski M, Fayers P, Visser MRM, Cull A, Hopwood P, Sanderman R (1996) The Rotterdam Symptom Checklist. Northern Centre for Healthcare Research (NCH), The Netherlands

11. Cheung YT, Lee HH, Chan A (2013) Exploring clinical determinants and anxiety symptom domains among Asian breast cancer patients. Support Care Cancer 21(8):2185-2194

12. Beck AT et al (1988) An inventory for measuring clinical anxiety: psychometric properties. J Consult Clin Psychol 56(6):893-897

13. Beck AT, Steer RA (1987) Manual for the Beck Anxiety Inventory. The Psychological Corporation, San Antonio

14. Grant M (2011) Beck Anxiety Inventory. In: Goldstein S, Naglieri J (eds) Encyclopedia of child behavior and development. Springer, New York, pp 215-217

15. Overcash J et al (2001) Validity and reliability of the FACT-G scale for use in the older person with cancer. Am J Clin Oncol 24(6):591-596

16. Cella DF et al (1993) The Functional Assessment of Cancer Therapy scale: development and validation of the general measure. J Clin Oncol 11(3):570-579

17. Chang VT et al (2000) Symptom and quality of life survey of medical oncology patients at a veterans affairs medical center. Cancer 88(5): $1175-1183$

18. Cheng KKF et al (2005) Measuring symptom prevalence, severity and distress of cancer survivors. Clin Eff Nurs 9(3-4):154-160

19. Stark $D$ et al (2002) Anxiety disorders in cancer patients: their nature, associations, and relation to quality of life. J Clin Oncol 20(14): 3137-3148

20. Jacobsen PB et al (2005) Screening for psychologic distress in ambulatory cancer patients. Cancer 103(7):1494-1502

21. Stanton AL (2006) Psychosocial concerns and interventions for cancer survivors. J Clin Oncol 24(32):5132-5137
22. Rehse B, Pukrop R (2003) Effects of psychosocial interventions on quality of life in adult cancer patients: meta analysis of 37 published controlled outcome studies. Patient Educ Couns 50(2):179-186

23. Zenz M et al (1995) Severe undertreatment of cancer pain: a 3-year survey of the German situation. J Pain Symptom Manag 10(3): 187-191

24. Deandrea $S$ et al (2008) Prevalence of undertreatment in cancer pain. A review of published literature. Ann Oncol 19(12):1985-1991

25. Pascual López A et al (2004) Systematic review of megestrol acetate in the treatment of anorexia-cachexia syndrome. J Pain Symptom Manag 27(4):360-369

26. Theobald DE (2004) Cancer pain, fatigue, distress, and insomnia in cancer patients. Clin Cornerstone 6(Supplement 1D):S15-S21

27. Cheng KKF, Lee DTF (2011) Effects of pain, fatigue, insomnia, and mood disturbance on functional status and quality of life of elderly patients with cancer. Crit Rev Oncol Hematol 78(2):127-137

28. Donovan KA, Jacobsen PB (2007) Fatigue, depression, and insomnia: evidence for a symptom cluster in cancer. Semin Oncol Nurs 23(2):127-135

29. Schaffer CB et al (2011) Efficacy and safety of nonbenzodiazepine hypnotics for chronic insomnia in patients with bipolar disorder. J Affect Disord 128(3):305-308

30. Najib J (2006) Eszopiclone, a nonbenzodiazepine sedative-hypnotic agent for the treatment of transient and chronic insomnia. Clin Ther 28(4):491-516

31. Westman G et al (1999) Megestrol acetate in advanced, progressive, hormone-insensitive cancer. Effects on the quality of life: a placebo-controlled, randomised, multicentre trial. Eur J Cancer 35(4):586-595

32. García VR, Juan O (2005) Megestrol acetate - probably less effective than has been reported! J Pain Symptom Manag 30(1):4

33. McMillan DC et al (1994) Effect of megestrol acetate on weight loss, body composition and blood screen of gastrointestinal cancer patients. Clin Nutr 13(2):85-89

34. Ron IG et al (2002) A low-dose adrenocorticotropin test reveals impaired adrenal function in cancer patients receiving megestrol acetate therapy. Eur J Cancer 38(11):1490-1494

35. Ozguroglu M et al (2006) Megestrol acetate induced hypoadrenalism with cushingoid phenotype in endometrial cancer. Gynecol Oncol 101(1):183

36. Stockheim JA et al (1999) Adrenal suppression in children with the human immunodeficiency virus treated with megestrol acetate. J Pediatr 134(3):368-370 